\title{
The Effect of Hydrogen on the Internal Friction of Deformed Vanadium
}

\author{
By Masahiro Koiwa*, Kazuhiko Shibata** \\ and Osamu Yoshinari***
}

\begin{abstract}
The internal friction of deformed vanadium has been measured in a torsion pendulum over a temperature range between -190 and $+300^{\circ} \mathrm{C}$. The oxygen and hydrogen contents are monitored for individual wire specimens by measuring the height of the oxygen Snoek peak and the position of the precipitation peak (or self-twisting) associated with the hydride precipitation.

It is concluded that the presence of hydrogen is essential for the appearance of the $\alpha$-peak.
\end{abstract}

(Received October 8, 1979)

\section{Introduction}

A low temperature relaxation peak termed the $\alpha$-peak ${ }^{(1)}$ has been reported by many investigators in $b c c$ refractory metals after plastic deformation. The peak has been suggested to be an "intrinsic" dislocation effect ${ }^{(2)}$, while some investigators have found that hydrogen plays an important role in the appearance of the $\alpha$-peak in vanadium ${ }^{(3)}$, niobium $^{(4)}$ and tantalum ${ }^{(5)}$. In the present paper is reported a detailed study of the $\alpha$-peak in vanadium. The metal vanadium is very reactive and usually contains a large amount of interstitial impurity atoms. It is difficult to avoid contaminations during annealing or handling of specimens. In this work, therefore, the concentration of oxygen and hydrogen has been monitored for individual specimen wires.

As reported in a preliminary note ${ }^{(6)}$, it is concluded that the presence of hydrogen is essential for the appearance of the $\alpha$-peak in vanadium.

\section{Experimental}

The vanadium used in this investigation was

* The Research Institute for Iron, Steel and Other Metals, Tohoku University, Sendai 980, Japan.

* Graduate School, Tohoku University, Sendai 980, Japan. Present address: TDK Electronics Co. Ltd., Tokyo 101, Japan.

*** Graduate School, Tohoku University, Sendai 980 , Japan. obtained from Nihon Gaishi Inc. in the form of granules $(99.8 \%$ pure). The chemical analysis of the raw material was given in a previous paper $^{(7)}$. From the ingot prepared by electron beam melting, wires $0.6 \mathrm{~mm}$ in diameter were made by swaging and cold drawing without intermediate annealings.

Specimen wires $70 \mathrm{~mm}$ in length were annealed with zirconium foils in an evacuated and sealed quartz tube for $2 \mathrm{~h}$ at, unless otherwise stated, $1100^{\circ} \mathrm{C}$. This annealing procedure (hereafter referred to as the zirconium treatment or anneal) is very effective to reduce the oxygen content in vanadium ${ }^{(7)(8)}$. The residual resistivity ratio of specimens thus prepared was typically about $190^{(7)}$, and the Vickers hardness (50 g load) was about 58. Some specimens were annealed by direct current heating at $1600^{\circ} \mathrm{C}$ for $25 \mathrm{~h}$ in a vacuum of $3 \times 10^{-6} \mathrm{~Pa}$ $\left(2 \times 10^{-8}\right.$ torr $)$; this annealing procedure will be referred to as the HV treatment. After this treatment, the Vickers hardness was about 130.

Hydrogen charging was made in either of two different ways. Controlled charging was performed by a cathodic charging technique at $0^{\circ} \mathrm{C}$. The electrolyte was a $0.1 \mathrm{~N} \mathrm{H}_{2} \mathrm{SO}_{4}$ solution and the current density was $100 \mathrm{~A} / \mathrm{m}^{2}$. Uncontrolled charging was made by simply dissolving zirconium foils attached to the surface, after the zirconium treatment, in a dilute HF solution; the amount of absorbed hydrogen can be controlled to some extent by changing the concentration of HF.

The internal friction was measured in an inverted torsion pendulum ${ }^{(7)}$ over a tempera-

1980 Vol. 21

Trans. JIM 
ture range from -190 to $+300^{\circ} \mathrm{C}$, usually at a heating rate of $1.25^{\circ} \mathrm{C} / \mathrm{min}$. A unique feature of the apparatus is that the measurement can be made both below and above room temperature without remounting the specimen. The oxygen content of a specimen can be estimated from the height of the Snoek peak $\left(\sim 180^{\circ} \mathrm{C}\right)$ before or after the measurement of internal friction at lower temperatures.

The measuring frequency is typically about $1.5 \mathrm{~Hz}$, and the strain amplitude in the maximum surface shear strain is $7 \times 10^{-6}$.

The zero point drift of the torsion pendulum was optically detected and recorded on a chart paper during measurements of internal frictions.

\section{Results and Discussion}

\section{Effect of oxygen on the $\alpha$-peak height}

Figure 1 shows the low temperature spectra of two specimens, $\mathrm{HV}$ annealed and $\mathrm{Zr}$ annealed, before and after deformation. The oxygen contents of the specimens are es-

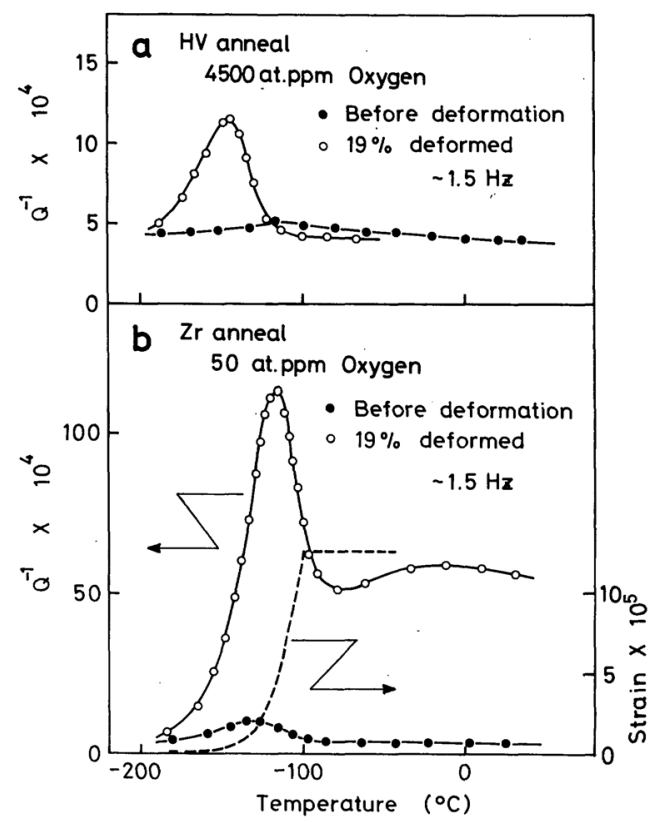

Fig. 1 Internal frictions before and after plastic deformation in specimens with high (a) and low (b) oxygen contents. The self-twisting is also shown for (b). timated as 4500 and 50 at.ppm from the Snoek peak height, by using the relation due to Hasson and Arsenault ${ }^{(9)}$,

$$
\text { [at.ppm oxygen] }=1.19 \times 10^{5} \cdot Q_{m}^{-1} \text {. }
$$

The specimens were torsionally deformed $19 \%$ in surface shear strain at room temperature. The $\alpha$-peak appears in both specimens, but in different strengths and at different temperatures:

$$
\begin{array}{r}
4500 \text { at.ppm O: } Q_{m}^{-1} \simeq 7.5 \times 10^{-4} \text { at }-145^{\circ} \mathrm{C} \\
50 \text { at.ppm } \mathrm{O}: Q_{m}^{-1} \simeq 81 \times 10^{-4} \text { at }-117^{\circ} \mathrm{C} .
\end{array}
$$

The effect of oxygen on the $\alpha$-peak has been reported by Chang and Wert ${ }^{(10)(11)}$, but is less drastic; the peak of $12 \times 10^{-4}$ in pure vanadium is reduced to $8 \times 10^{-4}$ by the addition of 3000 at.ppm $\mathrm{O}$.

\section{Effect of hydrogen on the $\alpha$-peak}

The content of hydrogen in vanadium can be sensitively estimated by monitoring the zero point drift of the pendulum, as first observed by Owen and $\mathrm{Scott}^{(12)}$; with the progress of the hydride precipitation or dissolution, a wire specimen twists by itself. In Fig. 1(b) is also plotted the self-twisting, (expressed in the surface shear strain) simultaneously recorded on measurements of internal friction. From the position of the knick, the hydrogen content is estimated to be about 150 at.ppm $\dagger$ in reference to the solubility data ${ }^{(13)}$. It has been found that the second zirconium treatment with new zirconium foils is effective in reducing the hydrogen content; in some occasions a single zirconium treatment reduces both oxygen and hydrogen contents. Details of the dehydrogenation study will be reported elsewhere ${ }^{(14)}$.

Figure 2 shows the result of a series of measurements on a specimen subjected to double zirconium treatments. The oxygen content is about 20 at.ppm. As shown in Fig. 2(a), the $\alpha$-peak does not appear after deformation. Then, the specimen is subjected to cathodic charging of hydrogen. The amount

$\uparrow$ In the previous paper ${ }^{(6)}$, we used the data by Owen and $\operatorname{Scott}^{(12)}$ to obtain 500 at.ppm. Since specimens used by Owen and Scott seem to contain a large amount of oxygen, we use the data by Chang and Wert ${ }^{(13)}$ in this paper. 


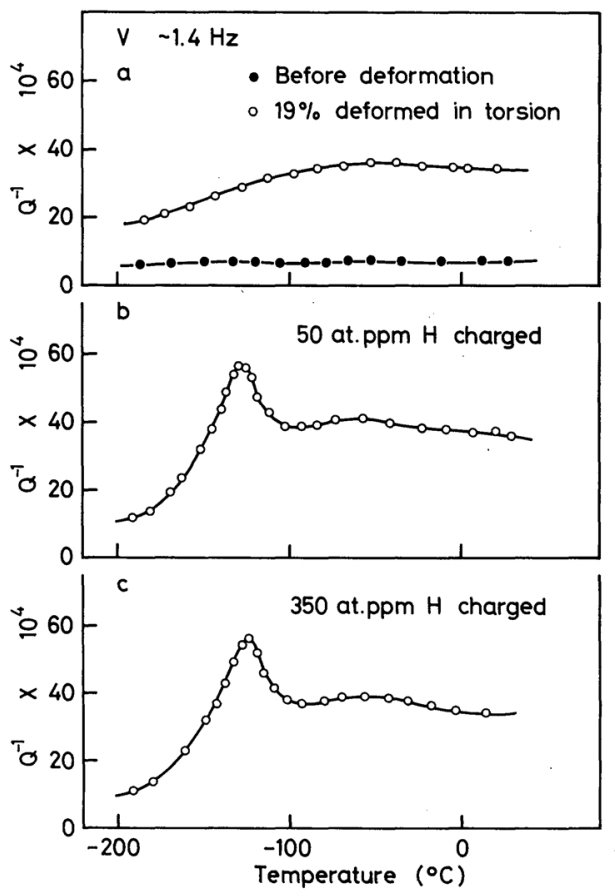

Fig. 2 The effect of hydrogen charging on the internal friction. The specimen is prepared by double zirconium treatments.

of hydrogen added was estimated from the increase in the electrical resistivity at $0^{\circ} \mathrm{C}$ by using the value $1.12 \times 10^{-8} \Omega \cdot \mathrm{m} /$ at. $\% \mathrm{H}^{(15)}$; numerical values of hydrogen contents thus determined may not be very reliable and should be regarded to give only a rough estimation, since cathodic charging of hydrogen was always made, in this work, on previously deformed specimens. After the addition of 50 at.ppm, the $\alpha$-peak appears at $-128^{\circ} \mathrm{C}$. The further addition to total 350 at.ppm does not change the general feature appreciably, except a small shift of the peak to $-124^{\circ} \mathrm{C}$. Figure 3 shows the internal friction, the frequency and the self-twisting, after the final charging to total 3260 at.ppm. The peak temperature for a frequency of about $1.4 \mathrm{~Hz}$ (open circles) is $-117^{\circ} \mathrm{C}$, which is the same as that for Fig. 1(b). In contrast to the curves in Fig. 2 for lower hydrogen contents, a shoulder is noticed at about $-60^{\circ} \mathrm{C}$, which is more clearly observed for the curve taken at a lower frequency of $0.54 \mathrm{~Hz}$. This is identified as the precipitation peak, which is known to be more dominant

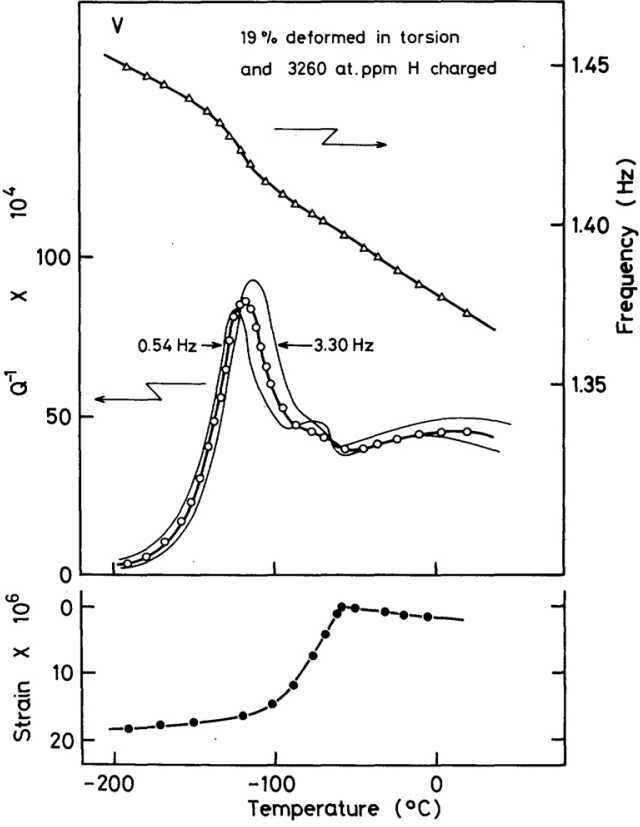

Fig. 3. The internal friction, frequency and selftwisting after hydrogen charging (continued from Fig. 2). Internal friction-temperature curves taken at frequencies of about 0.54 and $3.30 \mathrm{~Hz}$ are shown by thin lines.

for lower frequencies ${ }^{(7)}$. The self-twisting (the lower part of Fig. 3) shows a knick at about $-60^{\circ} \mathrm{C}$, corresponding to the precipitation peak.

In order to confirm the absence of the $\alpha$-peak in a "hydrogen-free" specimen, or more exactly, a specimen with an extremely low hydrogen content, the internal friction measurement was made on a specimen similar to that in Fig. 2(a), after successive deformations at room temperature. As shown in Fig. 4, the background value showed a monotonic increase with the increase in the degree of deformation, but no $\alpha$-peak was observed. After the final deformation of $38 \%$, the specimen was heated up to $300^{\circ} \mathrm{C}$. In the subsequent measurement a broad peak was observed at about $-55^{\circ} \mathrm{C}$, but no further studies were made on this peak.

The effect of hydrogen on the $\alpha$-peak has also been studied for specimens with high oxygen contents, as shown in Fig. 5. Four wire specimens subjected to the HV anneal were further annealed with zirconium foils for $2 \mathrm{~h}$ at different temperatures, 600, 800, 900 and 


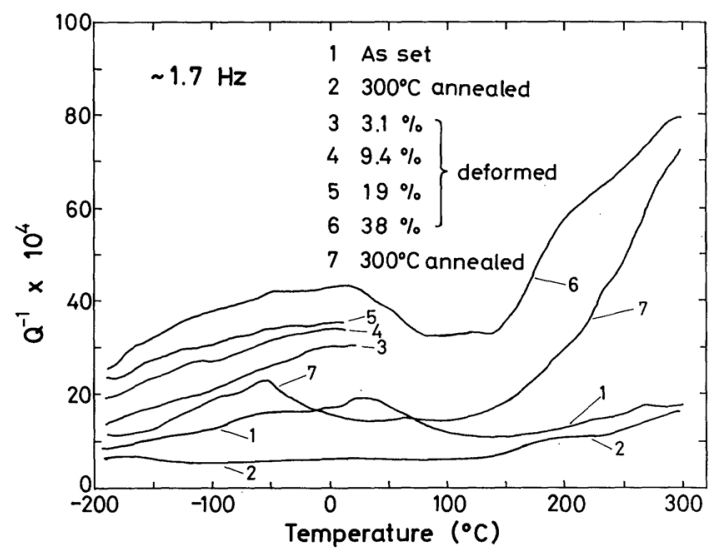

Fig. 4. The effect of deformation on the internal friction in a "hydrogen-free" specimen.

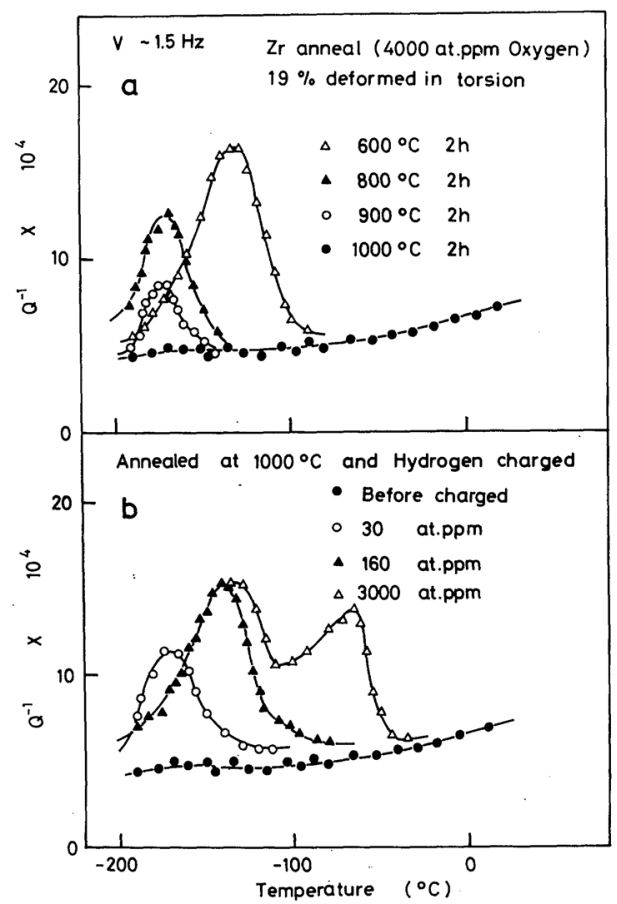

Fig. 5. The effect of dehydrogenation (a) and hydrogenation (b) on the internal friction in specimens with a high oxygen content.

$1000^{\circ} \mathrm{C}$. During these annealings, oxygen contents remain virtually unchanged $(\sim 4000$ at.ppm). The $\alpha$-peak is not observed in the specimen annealed at $1000^{\circ} \mathrm{C}$ and subsequently deformed $19 \%$, indicating the absence of hydrogen. This specimen was then subjected to successive charging of hydrogen up to 3000 at.ppm. The $\alpha$-peak appears at $-172^{\circ} \mathrm{C}(30$ at.ppm), $-140^{\circ} \mathrm{C}\left(160\right.$ at.ppm) and $-135^{\circ} \mathrm{C}$ (3000 at.ppm). The precipitation peak is also observed after the final charging of hydrogen.

It should be noted here that, for a given dislocation structure, the height of the $\alpha$-peak seems to saturate at a relatively low concentration of hydrogen $\dagger$ (see Figs. 2 and 5), which suggests that the height is limited by the amount of hydrogen in solid solution; the hydrogen solubility is less than 100 at.ppm ${ }^{(14)}$ at the temperature of the $\alpha$-peak $\left(\sim-120^{\circ} \mathrm{C}\right)$. The height of the $\alpha$-peak in hydrogen saturated (or hydrides containing) specimens would be larger for higher frequency measurements, since the peak shifts towards higher temperatures where the amount of hydrogen in solid solution is larger. Such a trend is in fact observed as seen in Fig. 3.

\section{Effect of the degree of deformation}

The effect of the degree of deformation on the height of the $\alpha$-peak has been studied on one specimen by measuring the internal friction after successive twisting up to total $31 \%$, as shown in Figs. 6 and 7. The specimen is the same as that for Fig. 1(b). The small peak observed before deformation may be the precipitation peak and/or the trace of the $\alpha$-peak introduced by handling. The peak height after subtraction of the background shows an initial rapid increase followed by a linear increase as a function of the degree of deformation (Fig. 7). The peak temperature remains almost constant at $-117 \pm 1^{\circ} \mathrm{C}$ throughout the whole range of strain. This dependence of peak position is consistent with observations on niobium ${ }^{(16)}$, but not with those on vanadium by Chang and Wert ${ }^{(10)(11)}$.

\section{Effect of the mode of deformation}

The height of relaxation peaks associated with dislocations may be dependent on the mode of plastic deformation ${ }^{(17)}$. Therefore, we have examined the effect of different modes of de-

$\uparrow \quad$ The larger peak height after charging 3260 at.ppm $\mathrm{H}$ (Fig. 3) in comparison to that with 350 at.ppm $\mathrm{H}$ (Fig. 2c) is considered to be due to the introduction of new dislocations associated with hydride precipitation. 


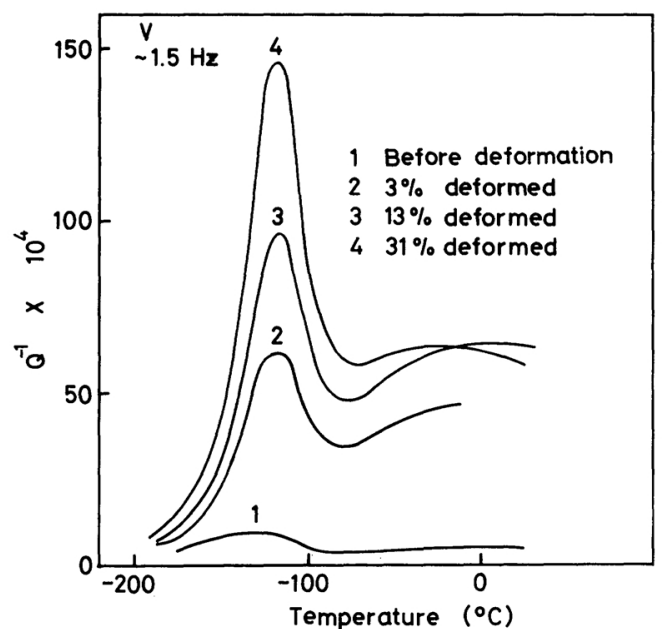

Fig. 6. The effect of increasing deformation in torsion on the internal friction. The specimen is the same as that in Fig. 1(b).

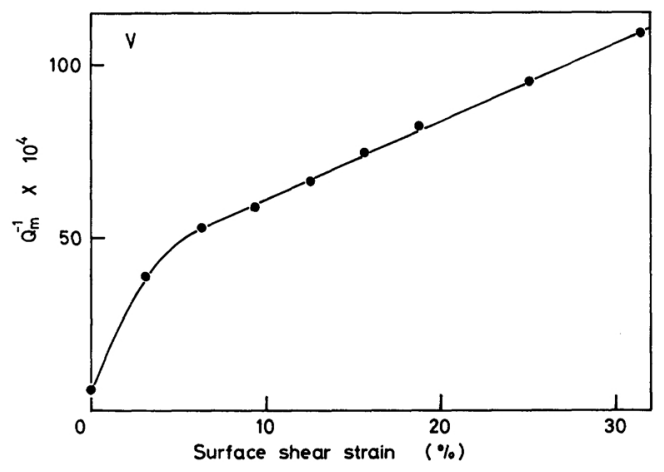

Fig. 7 The height of the $\alpha$-peak as a function of strain. The data are taken from Fig. 6.

formation on the $\alpha$-peak and also on the precipitation peak.

For measurements of internal friction so far described, zirconium foils attached to the surface of specimens after annealing at high temperatures were simply torn off mechanically, since any chemical treatments would introduce hydrogen into the specimen. In the case of the specimens to be described below, hydrogen is introduced by dissolving away zirconium foils in a dilute HF solution.

Figure 8 shows the effect of tensile deformation at room temperature for such a specimen. The hydrogen content is estimated, from the position of the precipitation peak, as about 8000 at.ppm. The tensile deformation does in-

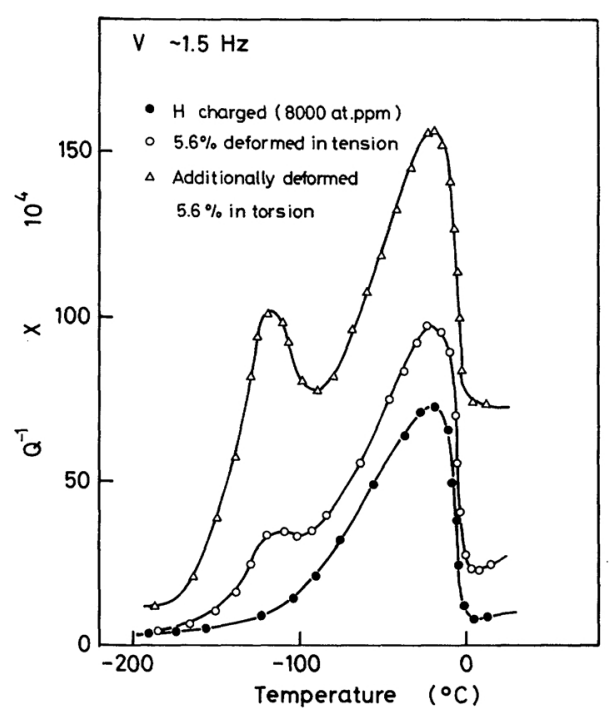

Fig. 8 The effect of tensile deformation and additional torsional deformation on the internal friction in a specimen of a low oxygen content.

troduce the $\alpha$-peak, but an additional torsional deformation strongly enhances the $\alpha$-peak. The background value of internal frictions becomes very large by deformation, but the height and the position of the precipitation peak are little affected.

Figure 9 compares the effect of deformation by drawing and by twisting on two different specimens with a similar hydrogen content of about 4500 at.ppm. The degree of drawing deformation is expressed in the reduction of the cross-sectional area. Although the changes in the background values are considerably different, the height of the $\alpha$-peak is nearly the same for the two modes of deformation.

\section{Annealing behaviour}

The effect of aging on the $\alpha$-peak in the specimen deformed $31 \%$ (curve 4 of Fig. 6) has been studied as shown in Fig. 10. Measurements of internal friction were made at successively higher temperatures, where the specimen was kept for $1 \mathrm{~h}$.

A similar study of aging has also been made on a specimen with a high oxygen content (5000 at.ppm) after 19\% deformation (Fig. 11). The self-twisting during internal friction measurements is also shown in this figure. Before deformation, the twisting is hardly observed. 


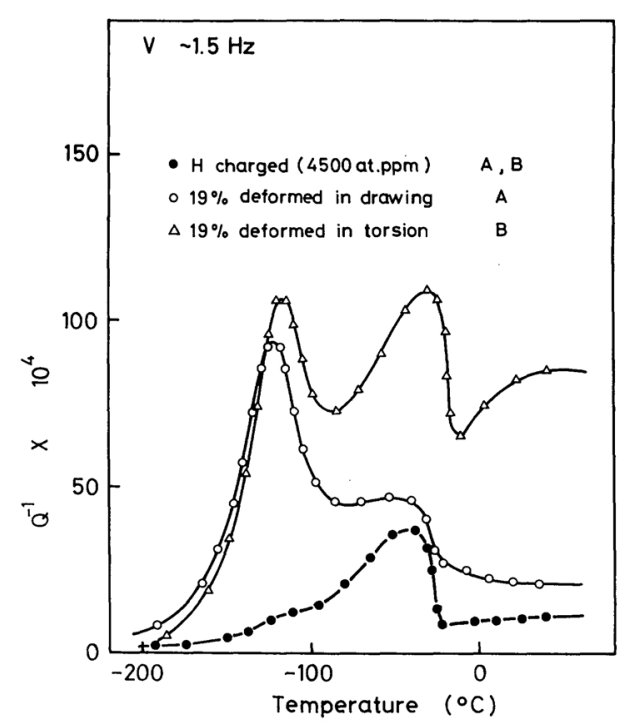

Fig. 9 Comparison of different modes of deformation; drawing (specimen A) and twisting (specimen B). Specimens are of low oxygen contents. The spectra before deformation are virtually the same for specimens $\mathbf{A}$ and $\mathbf{B}$.

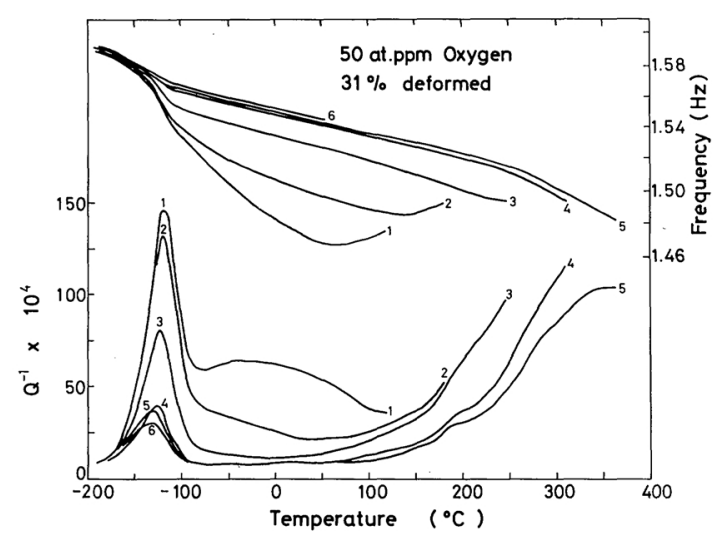

Fig. 10 The effect of aging on the internal friction and the frequency on a specimen with 50 at.ppm $O$. (continued from Fig. 6). Curve 1 is taken after deformation of $31 \%$. Maximum temperatures of measurements for successive runs are: $117^{\circ} \mathrm{C}(1)$, $180^{\circ} \mathrm{C}(2), 240^{\circ} \mathrm{C}(3), 309^{\circ} \mathrm{C}(4)$ and $360^{\circ} \mathrm{C}(5)$; curve 6 is taken after run 5 .

After deformation, a large twisting is observed with a knick at about $-100^{\circ} \mathrm{C}$. The twisting is clearly observed even after the anneal at $360^{\circ} \mathrm{C}$. It is added here that general features of selftwisting are similar for the specimen in Fig. 10.

The heights of the $\alpha$-peaks, after subtraction

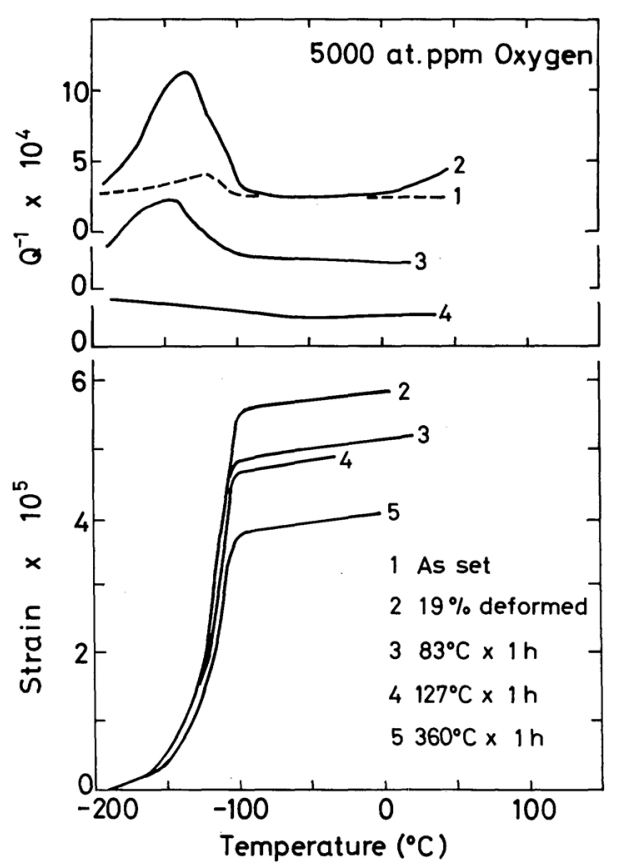

Fig. 11 The effect of aging on the internal friction and self-twisting in a specimen with 5000 at.ppm $O$.

of appropriate backgrounds, in Figs. 10 and 11 are shown as a function of annealing temperatures in Fig. 12. The complete disappearance of the $\alpha$-peak after annealing at $127^{\circ} \mathrm{C}$ for $1 \mathrm{~h}$ (Fig. 11) is reasonably understood as a result of oxygen diffusion and resultant pinning of dislocations; the number of jumps of oxygen atoms during $1 \mathrm{~h}$ at $127^{\circ} \mathrm{C}$ is estimated to be about 190 , using the parameters: $E=1.25 \mathrm{eV}$, $\tau_{0}=2.27 \times 10^{-15} \mathrm{~s}^{(18)}$.

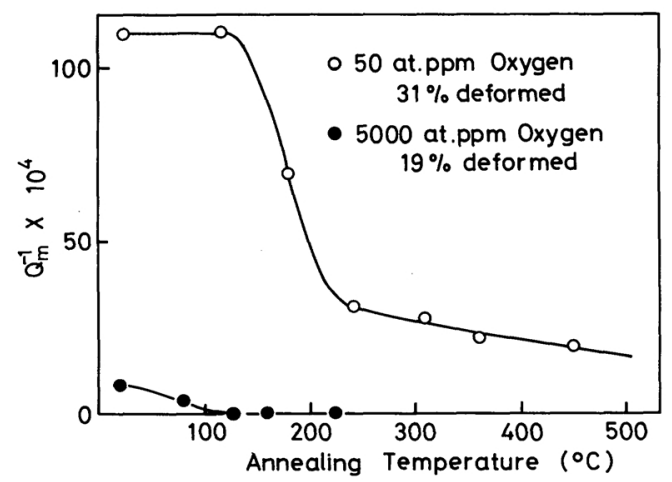

Fig. 12 The height of the $\alpha$-peak as a function of annealing temperatures. The data are taken from Figs. 10 and 11. 
The drastic decay of the peak in the specimen of a lower oxygen content in the temperature range between 100 and $200^{\circ} \mathrm{C}$ may be also ascribed to oxygen. For this specimen the $\alpha$ peak still exists in a considerable strength even after annealing at temperatures higher than $300^{\circ} \mathrm{C}$. At this stage, the oxygen Snoek peak also exists, which indicates the presence of oxygen in the matrix (see curves 4 or 5 in Fig. $10)$. This may suggest that the oxygen concentration along dislocations in equilibrium with the matrix concentration of about 50 at.ppm is low enough and so the mean loop length of dislocations is large enough to contribute to the $\alpha$-relaxation. The peak temperatures are also reduced with the progress of annealing, which is consistent with observations on niobium and molybdenum ${ }^{(19)}$.

\section{Activation energy}

The activation energy of the $\alpha$-peak has been determined by the peak shift method, as shown in Fig. 13. For this experiment, two specimens of different oxygen contents were used. Open circles are taken from the data shown in Fig. 3; the oxygen content is about 20 at.ppm, and the hydrogen content is 3260 at.ppm. Similar measurements were also made for the specimen

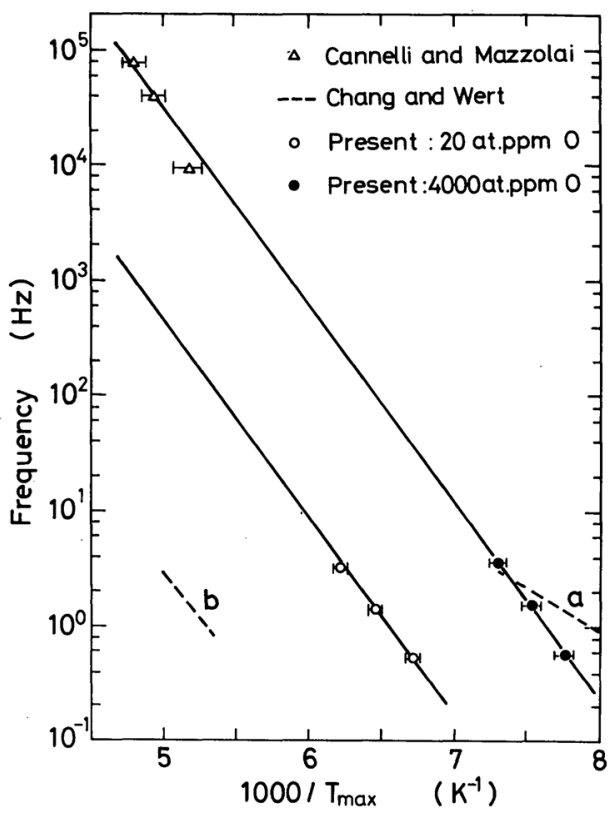

Fig. 13 Arrhenius plots for the $\alpha$-peak. shown in Fig. 1(b) $(\sim 150$ at.ppm $H)$; the peak temperatures are virtually the same as those in Fig. 13, and are not shown here.

Filled circles are for the specimen of a high oxygen content of 4000 at.ppm (Fig. 5b) after the hydrogen charging of 3000 at.ppm. It should be noted that the straight line drawn through the present three data points also passes the data points at higher frequencies $(\sim 10 \mathrm{kHz})$ by Cannelli and Mazzolai ${ }^{(3)}$; the oxygen contents of their specimens are estimated to be about 6000 at.ppm in reference to the work by Chang and Wert ${ }^{(13)}$. By combining the two sets of data points, the activation parameters $\tau_{0}$ and $E$ in $\tau=\tau_{0} \exp (E / k T)$ are determined as

$$
\begin{aligned}
& \tau_{0}=1.5 \times 10^{-14} \mathrm{~s},\left(v=\tau_{0}^{-1}=6.7 \times 10^{13} \mathrm{~s}^{-1}\right) \\
& E=0.34 \pm 0.02 \mathrm{eV} .
\end{aligned}
$$

For specimens with a low oxygen content, only those data in a narrow frequency range are available, and one cannot expect a great accuracy;

$$
\begin{aligned}
& \tau_{0}=4.7 \times 10^{-13 \pm 2} \mathrm{~s} \quad\left(\nu=2.1 \times 10^{12 \pm 2} \mathrm{~s}^{-1}\right), \\
& E=0.35 \pm 0.07 \mathrm{eV} .
\end{aligned}
$$

The straight line was just drawn in parallel with that for the high oxygen contents.

It seems that the addition of oxygen does not affect the activation energy, but increases the attempt frequency $v$. A similar trend has been reported for niobium ${ }^{(16)}$.

For the sake of comparison, some of the results by Chang and Wert ${ }^{(10)}$ are also shown by the broken lines; the line $a$ is for "the $\alpha$-peak" e.g. in a specimen without intentional hydrogen charging, and the line $b$ for "Type I Snoek-Köster peak" in a specimen with 450 at.ppm hydrogen. These results will be discussed later.

\section{Peak shape}

After plastic deformation, a high background is usaually observed which increases with temperature. The background decays faster than the $\alpha$-peak with annealing, as seen in Fig. 10. Therefore, we examined the shape of the $\alpha$-peak after annealing at $180^{\circ} \mathrm{C}$ (curve 3 in Fig. 10). The internal friction and the frequency, 
after subtraction of appropriate backgrounds, are plotted as a function of reciprocal temperatures in Fig. 14. The peak is broader than a single relaxation peak for the activation energy $0.35 \mathrm{eV}$. The magnitude of the $\Delta M$ effect is large $\left(\Delta G / G=3.38 \times 10^{-2}\right)$ in comparison to the height of the internal friction peak $\left(2 Q_{m}^{-1}=1.40 \times 10^{-2}\right)$. These two facts imply the distribution in the relaxation time. The peak is more broader at the low temperature side, which may be an indication of a subsidiary peak, as in the case of niobium ${ }^{(20)}$.

\section{Comparison with previous work}

The internal friction of vanadium has been studied previously by Chang and Wert ${ }^{(10)(11)}$, and we shall compare the present result with theirs. The most remarkable difference is the height of the $\alpha$-peak; it is almost an order of magnitudes larger in our experiments. At first it was suspected that the difference in the mode of deformation might be responsible. They deformed specimens by swaging, while we deformed mostly by twisting. Although the tensile deformation seems less effective (Fig. 8), the drawing deformation, which is also a severe mode of deformation like swaging,

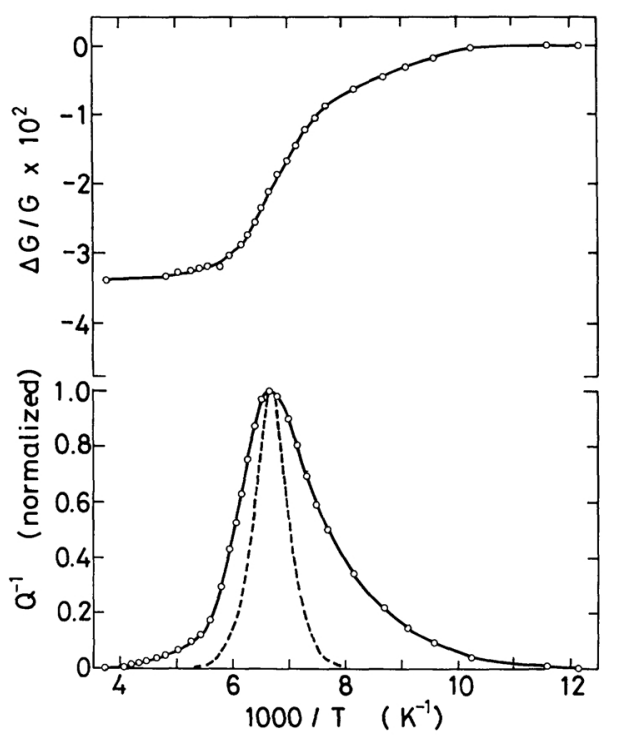

Fig. 14 The shape of the $\alpha$-peak and the associated $\Delta M$ effect. The data are taken from curve 4 in Fig. 10. The broken line is the single relaxation peak calculated for the activation energy $0.35 \mathrm{eV}$. introduced a large $\alpha$-peak (Fig. 9).

This and other differences in the behaviour of the internal friction peaks in the two experiments are reasonably understood, if we assume that their "pure vanadium" contained a fairly large amount of oxygen. They did not state the oxygen contents of the starting material or the final wire specimens. Their outgassing treatment of heating two hours at $1450^{\circ} \mathrm{C}$ in a vacuum of $10^{-6} \operatorname{torr}^{(10)}$ does not seem sufficient for the removal of oxygen, in reference to the result of the kinetic study of deoxygenation of vanadium by $\mathrm{Hörz}^{(21)}$. The smallness of the hydride precipitation peak (compare Figs. 8 and 9 of this paper and Fig. 12 of Ref. 10) is also consistent with the assumption of a high oxygen content.

In the present experiment, the peak temperature of the $\alpha$-relaxation shows a variation with the contents of hydrogen and oxygen, but is always lower than $-115^{\circ} \mathrm{C}$ for frequencies of about $1.5 \mathrm{~Hz}$. In the case of the experiment by Chang and Wert ${ }^{(10)}$, the peak temperature of the $\alpha$-peak or the type I and II Snoek-Köster peak is as high as $-80^{\circ} \mathrm{C}$ in some specimens (e.g. Fig. 8 of Ref. 10) for nearly the same frequency. It is likely that such a peak may be a superposition of the true $\alpha$-peak and the precipitation peak, the position and the magnitude of which change widely with the concentration of hydrogen.

The presence of the $\alpha$-peak in their "pure vanadium" may be attributed to residual hydrogen. A few ten ppm hydrogen is sufficient to cause the $\alpha$-peak to appear and such a small amount of hydrogen may be present in any specimens $^{(22)}$ unless a special care is undertaken; the degassing in ultra high vacuum does not necessarily assure the absence of hydrogen in the specimen at the time of internal friction measurements.

The result of the present experiment supports the view of Cannelli and Mazzolai ${ }^{(3)}$ that the $\delta$-peak is the intrinsic dislocation effect and that the $\alpha$-peak is a combined effect of dislocations and hydrogen atoms. A similar picture has also been proposed for iron by Takita and Sakamoto $^{(23)}$. As to the mechanism of the $\alpha$-peak, the Schoeck model ${ }^{(24)}$ has been considered to be reasonable. In this connection, 
it seems interesting to compare the present result on $\mathrm{V}-\mathrm{H}$ alloys to that on $\mathrm{V}-\mathrm{D}$ alloys ${ }^{(7)}$. A peak introduced by thermal cycling or by plastic deformation in a V-D alloy, which is now identified as the $\alpha$-peak, appears at similar temperatures $\left(\sim-120^{\circ} \mathrm{C}\right)$ to those in $\mathrm{V}-\mathrm{H}$ alloys. The diffusivities of $H$ and $D$ in vanadium is known to be different considerably ${ }^{(25)}$. If the relaxation times of the $\alpha$-peak is related to the diffusion constant of hydrogen or deuterium as is the case of the Schoeck model, the peaks should appear at fairly different temperatures. Further studies are in progress to clarify the nature of the $\alpha$-peak.

\section{Summary and Conclusions}

(1) The internal friction of deformed vanadium has been measured in a torsion pendulum between $-190^{\circ} \mathrm{C}$ and $+300^{\circ} \mathrm{C}$. The oxygen and hydrogen contents of individual specimens were monitored by measuring the Snoek peak height and by observing the position of the precipitation peak or self-twisting due to hydride precipitation.

(2) Both the oxygen and hydrogen contents in vanadium can be reduced by annealing with zirconium foils.

(3) The $\alpha$-peak in vanadium requires the presence of hydrogen.

(4) The addition of oxygen greatly suppresses the height of the $\alpha$-peak, and lowers the peak temperature.

(5) The $\alpha$-peak completely disappears after annealing at $127^{\circ} \mathrm{C}$ in the specimen with a high oxygen content (5000 at.ppm), but it still remains in a considerable strength after annealing at $450^{\circ} \mathrm{C}$ in the specimen with a low oxygen content (50 at.ppm).

(6) The activation energies and attempt frequencies are:

$$
\begin{array}{cl}
20 \text { at.ppm } O & E=0.35 \pm 0.07 \mathrm{eV} \\
& v=2.1 \times 10^{12 \pm 2} \mathrm{~s}^{-1} \\
4000 \text { at.ppm } O & E=0.34 \pm 0.02 \mathrm{eV} \\
& v=6.7 \times 10^{13} \mathrm{~s}^{-1}
\end{array}
$$

(7) A further insight as to the mechanism of the $\alpha$-peak may be obtained by comparing the effect of the addition of hydrogen and deuterium.

\section{Acknowledgements}

The authors wish to express their thanks to Professor M. Hirabayashi for continued interests and encouragements. Their thanks are also due to Mr. S. Tanaka for his help in the hydrogen charging of specimens. They also wish to thank Messers. S. Ono, K. Wako and $\mathrm{K}$. Obara for helps in the preparation of specimens.

This work is partly supported by the Grant in Aid for Scientific Research from the Ministry of Education.

\section{REFERENCES}

(1) R. H. Chambers: Physical Acoustics, Ed. by W. P. Mason, Academic Press, New York (1966), Vol. III A, p. 123.

(2) A. Seeger and C. Wüthrich: Nuovo Cimento, 33B (1976), 38.

(3) G. Cannelli and F. M. Mazzolai: J. Phys. Chem. Solids, 31 (1970), 1913.

(4) G. Ferron, M. Quintard and P. Mazot: Scripta Met., 12 (1978), 623.

(5) F. M. Mazzolai and M. Nuovo: Solid State Comm., 7 (1969), 103.

(6) K. Shibata, M. Koiwa and O. Yoshinari: Trans. JIM, 19 (1978), 491.

(7) O. Yoshinari, M. Koiwa, H. Asano and M. Hirabayashi: Trans. JIM, 19 (1978), 171.

(8) K. Abe, K. Toma, H. Yoshinaga and S. Morozumi: J. Less-Common Metals, 23 (1971), 213.

(9) D. F. Hasson and R. J. Arsenault: Scripta Met., 5 (1971), 75.

(10) H. Y. Chang and C. A. Wert: Ber. Bunsenges. Phys. Chem., 77 (1973), 42.

(11) H. Y. Chang and C. A. Wert: Proc. Fifth Int. Conf. on Internal Friction and Ultrasonic Attenuation in Crystalline Solids, Ed. by D. Lenz and K. Lücke, Springer-Verlag (1975), Vol. I, p. 259.

(12) C. V. Owen and T. E. Scott: Met. Trans., 3 (1972), 1715.

(13) H. Y. Chang and C. A. Wert: Acta Met., 21 (1973), 1233.

(14) M. Koiwa and K. Shibata: to be published.

(15) D. G. Westlake: Trans. Met. Soc. AIME, 239 (1967), 1341.

(16) M. F. Amateau, T. E. Mitchell and R. Gibala: Phys. Status Solidi, 36 (1969), 407.

(17) M. Koiwa and R. R. Hasiguti: Acta Met., 11 (1963), 1215.

(18) G. Hörz: Z. Metallk., 59 (1968), 712.

(19) R. Gibala, M. K. Korenko, M. F. Amateau and T. E. Mitchell: J. Phys. Chem. Solids, 31 (1970), 1889. 
(20) R. Klam, H. Schultz and H.-E. Schaefer: Acta Met., 27 (1979), 205.

(21) G. Hörz: Z. Metallk., 60 (1969), 50.

(22) E. Lang and J. Bressers: Z. Metallk., 67 (1976), 66.

(23) K. Takita and K. Sakamoto: Scripta Met., 10
(1976), 399.

(24) G. Schoeck: Acta Met., 11 (1963), 617.

(25) J. Völkl and G. Alefeld: Hydrogen in Metals, Ed. by G. Alefeld and J. Völkl, Springer-Verlag, Berlin (1978), Vol. I, p. 321. 